\title{
Evaluación del tiempo en la recuperación de larvas de nematodos a partir de un coprocultivo
}

\author{
Evaluation of time for recovery of nematode larvae from a coproculture
}

Leandro-Picado A, Calderón-Sancho A, Alfaro-Mora R. Evaluación del tiempo en la recuperación de larvas de nematodos a partir de un coprocultivo. Rev Colombiana Cienc Anim. Recia. 2020; 12(1):e744. DOI: https://doi.org/10.24188/recia.v12.n1.2020.744

Universidad de Sucre, Colombia

Los autores permiten a RECIA reimprimir el material publicado en él. En caso de que un autor quiera traducir o usar una publicación parcial o completa de nuestro Diario, el autor debe obtener un permiso por escrito del editor de la revista.

Copyright (C) 2020. El (los) autor (es), Revista Colombiana de Ciencia Animal - RECIA. 2020. Este es un artículo de acceso abierto distribuido bajo los términos de Creative Commons Attribution 4.0 (https://creativecommons.org/licenses/by-nc-sa/4.0/), El uso, distribución o reproducción está permitido, siempre que se acrediten al autor original y al propietario del copyright y que se cite la publicación original en esta revista, de acuerdo con la práctica académica aceptada. No se permite el uso, distribución o reproducción que no cumpla con estos términos. 


\title{
Evaluación del tiempo en la recuperación de larvas de nematodos a partir de un coprocultivo
}

\author{
Evaluation of time for recovery of nematode larvae from a coproculture
}

Andrea Leandro-Picado* Lic.

Universidad Latina de Costa Rica. Escuela de Farmacia. San José.

Costa Rica.

anleapic@gmail.com

(iD https://orcid.org/0000-0003-3697-0116

Alexander Calderón-Sancho. Lic.

Universidad Latina de Costa Rica. Escuela de Farmacia. San José.

Costa Rica.

alexandercalderon01@gmail.com

(D) https://orcid.org/0000-0002-7924-3392

Ramsés Alfaro-Mora. M.Sc.

Universidad Latina de Costa Rica. Dirección de Investigación. Escuela de Farmacia. San José, Costa Rica

ramses.alfaro@ulatina.net

(i) https://orcid.org/0000-0002-7331-9405
DOI: https://doi.org/10.24188/recia.v12.n1.2020.744

\section{RESUMEN}

Las infecciones producidas por nematodos gastrointestinales representan un problema de gran importancia para la salud en bovinos, además de afectar la economía de los ganaderos e industrias productoras. Se simuló las condiciones necesarias para el desarrollo de los huevos de los nematodos contenidos en las heces del bovino a través de un coprocultivo en aserrín de laurel (Laurus nobilis). En esta investigación se pretendió evaluar diferencias en el tiempo de recuperación de larvas de nematodos a partir del coprocultivo. En la metodología se realizó la técnica de flotación para la identificación de huevos de nematodos y el McMaster para contabilizar la cantidad de huevos por gramo de heces y la cantidad de larvas recuperadas del coprocultivo a los diferentes tiempos. El análisis de los resultados se realizó con el software Minitab versión 19, y se aplicó un ANOVA con un análisis post hoc de Tukey. Como resultado, se estableció que existe una diferencia significativa, de importancia, en el conteo de nematodos cuando estos se recolectan a las 3 horas.

Palabras Clave: Rumiante, nematodos, heces, flotación, Laurus nobilis, ANOVA.

\begin{abstract}
Gastrointestinal nematode infections represent a problem for the health of cattle, as well as affecting the economics of farmers and producing industries. The necessary conditions for the development of the eggs of the nematodes, contained in the feces of the bovine, were simulated using a coproculture in sawdust of laurel (Laurus nobilis). In this investigation it was tried to evaluate differences in the recovery time of nematode larvae from the coproculture. In the methodology, the flotation technique was used to identify nematode eggs and the McMaster to count the amount of eggs per gram of feces and the amount of larvae recovered from the coproculture at different times. The analysis of the results was performed with the Minitab software version 19, and an ANOVA was applied with a post hoc analysis of Tukey. It was established that exist a significant difference in the nematode count when they are collected at 3 hours.
\end{abstract}

Keywords: Rumiants, nematodes, feces, flotation, Laurus nobilis, ANOVA

Como citar (Vancouver).

Leandro-Picado A, Calderón-Sancho A, Alfaro-Mora R. Evaluación del tiempo en la recuperación de larvas de nematodos a partir de un coprocultivo. Rev Colombiana Cienc Anim. Recia. 2020; 12(1):e744. DOI: https://doi.org/10.24188/recia.v12.n1.2020.744 


\section{INTRODUCCIÓN}

La técnica del coprocultivo se utiliza para promover la maduración y eclosión de los huevos de nematodos, y la evolución de las larvas hasta el tercer estadio, la fase infestante (L3) (1). Para que esta técnica sea posible necesita de una humedad, oxigenación y temperatura adecuada (2). Además, es importante utilizar un sustrato que emule las condiciones del suelo, como lo es la madera, y que esta no sea tóxica para las larvas, tal es el caso del aserrín de laurel (Laurus nobilis) (3).

Los coprocultivos nos permiten identificar de mejor manera la identidad de los nematodos gastrointestinales que causan serias infecciones en los rumiantes (4). Esto representa un problema en el nivel mundial, en especial a los bovinos, debido a que la productividad del hospedador se ve afectada al causar reducciones en las tasas de crecimiento, fecundidad, así como un incremento en la mortalidad (5).

Las infecciones por nematodos son más comunes en animales de corta edad, debido a que su sistema inmunológico no está suficientemente desarrollado (6). En la literatura es común encontrar que los nematodos que se reportan principalmente en rumiantes son: Cooperia, Trichostrongylus, Haemonchus, Oesophagostomum, Ostertagia y Nematodirus (7).

Por otra parte, no se han encontrado estudios donde se hable acerca de cómo afecta la variación del tiempo en la obtención de nematodos gastrointestinales a partir de un coprocultivo en heces de bovinos. Por ese motivo, este estudio evaluó el tiempo en la recuperación de larvas de nematodos a partir de un coprocultivo con aserrín de laurel.

\section{MATERIALES Y MÉTODOS}

Se identificó un bovino con alta carga de huevos de nematodos, mediante el método de flotación con solución hipersaturada de sacarosa (8). Luego se preparó un coprocultivo, dentro de un recipiente de vidrio, con $10 \mathrm{~g}$ de heces, $10 \mathrm{~g}$ de aserrín de laurel (Laurus nobilis) y se adicionó aproximadamente $40 \mathrm{ml}$ de agua destilada, se tapó con papel aluminio perforado (Figura 1A), y se dejó reposar por 8 días a $27^{\circ} \mathrm{C}$. Posteriormente, se le adicionan cantidad suficiente de agua destilada a $37^{\circ} \mathrm{C}$ hasta que se forma un menisco con forma convexa, dos horas después se coloca una placa de Petri invertida sobre el recipiente y se le dio vuelta.

Se adicionó $16 \mathrm{~mL}$ de agua destilada a $37^{\circ} \mathrm{C}$ en el exterior de la placa, asegurándose cubrir los alrededores del recipiente (Figura 1B). Se mantuvo en reposo y el procedimiento se evaluó por triplicado en los tiempos de 0.5, 1, 2, 3 y 4 horas. Se realizó la recolecta del líquido externo a cada una de las muestras realizadas, se trasladaron a un tubo de ensayo que se mantuvo a $4{ }^{\circ} \mathrm{C}$ durante una hora. Pasado este tiempo se tomó $0.4 \mathrm{~mL}$ del fondo del tubo y se mezcló con una gota de lugol para ser analizado utilizando un McMaster como instrumento para realizar el conteo de larvas (Figura 1C) (9). Además, para el ensayo propuesto se evaluó a las 2 horas la especificidad comparando el medio de cultivo con heces vs uno sin heces, y la precisión a través de 6 repeticiones. El análisis de los resultados se realizó con el software Minitab versión 19, y se aplicó un ANOVA con un análisis post hoc de Tukey (10).
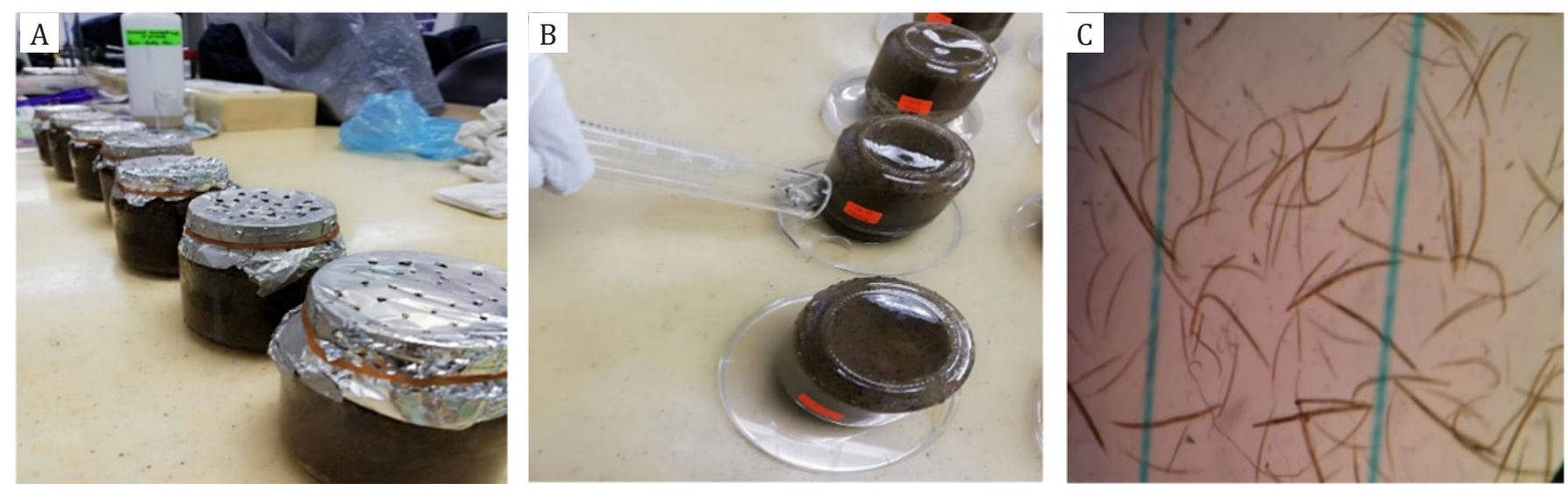

Figura 1. (A) Coprocultivos en envases de vidrio cubiertos con papel aluminio perforado. (B) Envase invertido sobre placa de Petri cubierta con agua destilada como medio de obtención de larvas. (C) Larvas en estadio L3 observadas a través del microscopio utilizando la cámara de McMaster. 


\section{RESULTADOS}

El animal del que se tomaron las muestras para el estudio tenía una edad de 4 meses y un conteo de 10050 huevos por gramo de heces, donde se encontró únicamente la especie Trichostrongylus sp., que coincide con las larvas obtenidas luego de que se realizara el cultivo. En la tabla 1 se muestran los resultados de la especificidad del método de cultivo, a través del uso de aserrín de laurel (Laurus nobilis).

Tabla 1. Datos del crecimiento de larvas comparando un medio de cultivo con y sin muestra, para evaluar la especificidad.

\begin{tabular}{cccccc}
\hline Medio de Cultivo & Cantidad de larvas en 2 horas & Promedio & Desviación Estándar \\
\hline Aserrín + Agua & $\mathrm{C}_{1}$ & $\mathrm{C}_{2}$ & $\mathrm{C}_{3}$ & $\overline{\mathrm{X}}$ & $\mathrm{SD}$ \\
Aserrín + Heces + Agua & 0 & 0 & 0 & 0 & 0 \\
\hline
\end{tabular}

Simbología: C representa el número del coprocultivo.

Al realizar diferentes repeticiones de la misma muestra se puede observar que la desviación estándar para la técnica resulta ser amplia, según se muestra en la tabla 2.

Tabla 2. Precisión del coprocultivo según número de larvas eclosionadas a diferentes tiempos.

\begin{tabular}{ccccccccc}
\hline Cultivo & \multicolumn{4}{c}{ Cantidad de larvas en 2 horas } & & Promedio & Desviación Estándar \\
\hline Aserrín + Heces+ Agua & $\mathrm{C}_{1}$ & $\mathrm{C}_{2}$ & $\mathrm{C}_{3}$ & $\mathrm{C}_{4}$ & $\mathrm{C}_{5}$ & $\mathrm{C}_{6}$ & $\bar{X}$ & $\mathrm{SD}$ \\
& 2 & 3 & 5 & 3 & 4 & 6 & 3.83 & 1,47 \\
\hline
\end{tabular}

Simbología: C representa el número del coprocultivo.

Se determinó la variación en el conteo de larvas de nematodos a distintos tiempos de recolecta posterior al cultivo, como se muestra en la figura 2. A las 3 horas se recuperó la mayor cantidad de larvas de Trichostrongylus sp. mostrando una diferencia significativa con respecto a los otros tiempos ensayados. El conteo de nematodos a las 3 horas mostró un porcentaje aparente de eclosión del $23 \%$.
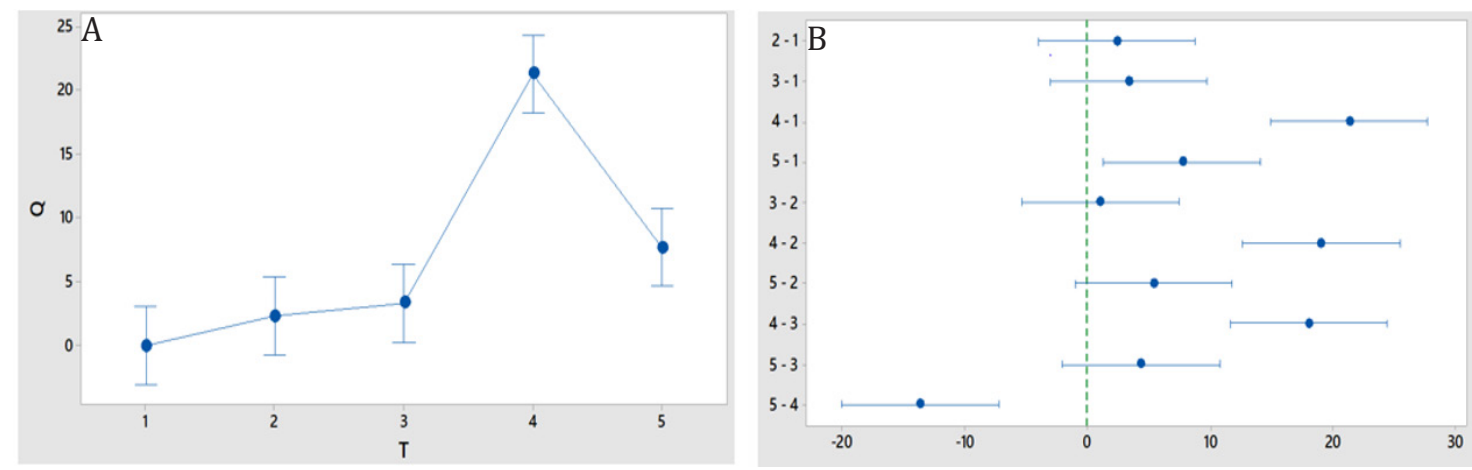

Figura 2. (A) Gráfica de intervalos de Q (cantidad de larvas) con respecto a $\mathrm{T}$ (Tiempo) a 95\% IC para la media. $1=0.5$ horas, $2=1$ hora, $3=2$ horas, $4=3$ horas, $5=4$ horas. (B) ICs simultáneos de 95\% de Tukey. Diferencias de las medias para Q (cantidad de larvas).

\section{DISCUSIÓN}

Se evaluó la especificidad de los datos del crecimiento de larvas al comparar un medio de cultivo con y sin muestra de heces, y se demuestra que hay una correcta especificidad de la técnica con el uso de aserrín de laurel, por lo que se puede decir que el aserrín de laurel (Laurus nobilis) se relaciona con falsos positivos, por lo tanto, no es un factor que afecte en los resultados obtenidos en los cultivos $(3,11)$. 
Para evaluar la precisión del estudio se elaboraron 6 cultivos como se muestra en la tabla 2. Esta representa la reproducibilidad que tiene la investigación o en otras palabras la homogeneidad que tiene la muestra a la hora de realizar los coprocultivos. Para efectos de la precisión, el método resulta generar una desviación estándar bastante considerable entre los diferentes ensayos a un mismo tiempo de espera (12). Pero esto podría ser consecuencia de pequeñas diferencias de minutos entre los tiempos de recolecta a las 2 horas, ya que como se podrá observar una diferencia en los tiempos de 60 minutos marca un importante cambio.

Luego de invertir el frasco con la placa Petri y añadirle agua alrededor de esta, el tiempo que se espera para recoger el sobrenadante es crucial ya que las larvas migran en un tiempo determinado. Por este motivo, se evaluó el ensayo por medio del método de análisis de exactitud donde se establecieron los tiempos indicados en la figura 2 (13). Haciendo uso del programa estadístico Minitab 19 y aplicando la prueba ANOVA de un factor con comparaciones múltiples post hoc en Tukey, se observó un incremento en el número de larvas de Trichostrongylus sp. a partir de una hora. Llegadas las tres horas se obtuvo un pico considerable de larvas en donde el conteo de estas era muy superior al de 2 horas. Por otra parte, un tiempo de espera de cuatro horas dio resultados similares a los de dos horas. Este último aspecto podría ser explicado por una situación en la que las larvas que ya han migrado al exterior, posterior a un tiempo de 3 horas intenten retornar a la zona de donde salieron, debido a la disminución de la temperatura en el medio externo. Otra posibilidad de este cambio podría estar relacionada a la vitalidad de la larva posterior a las 3 horas, lo cual deja claro que no es propicio una espera superior a ese tiempo, ya que los resultados no serían adecuados, mientras que la mayor recolecta se obtiene en ese punto máximo, y la diferencia con respecto a los otros tiempos es de significancia estadística. Para finalizar es importante señalar que los nematodos gastrointestinales en general poseen una buena resistencia a las temperaturas cálidas como frías, lo cual hace que el método utilizado y en los tiempos propuestos no afecte de mayor manera los resultados.

\section{Conflicto de Intereses}

Los autores no reportan conflictos de interés

\section{REFERENCIAS}

1. Angulo F, Molero M, Escalona F, Muñoz J, Ramírez R. Prevalencia y dinámica de hpg mensuales de fasciola hepática y otros helmintos en un rebaño bovino de una zona inundable tropical. Revista Científica. 2007; 17(2):111-116. http://ve.scielo.org/scielo.php?script=sci arttext\&pid=S0798-22592007000200002

2. Fiel C, Steffan P, Ferrey D. Diagnóstico de las parasitosis más frecuentes de los rumiantes. diagnóstico de las parasitosis más frecuentes de los rumiantes: Técnicas de laboratorio e interpretación de resultados. $1^{\circ}$ edición. Buenos Aires, Argentina. 2015. http://www.aavld.org.ar/publicaciones/Manual\%20Diagnostico\%20final.pdf

3. Álvarez C, Hernández J, WingChing R. Eficacia de aserrines para inhibir el desarrollo in vitro de larvas de parásitos gastrointestinales de ovinos. eficacia de aserrines para inhibir el desarrollo in vitro de larvas de parásitos gastrointestinales de ovinos. Agronomía Costarricense 2007; 31(1): 71-75. https://revistas.ucr.ac.cr/index.php/ agrocost/article/view/6821/6508

4. Ensuncho C, Castellano A, Maza L, Bustamante M, Vergara O. Prevalencia y grado de infección de nematodos gastrointestinales en ovinos de pelo en pastoreo de cuatro municipios de Córdoba, Colombia. Revista Científica. 2014; 16(5):414-420. https://www.redalyc.org/pdf/959/95932260005.pdf

5. Barrabí M, Arece J. Actividad antihelmíntica in vitro de extracto acuoso de hojas y semillas de Neem (Azadirachta indica A. Juss). I. Inhibición de la eclosión de huevos y del desarrollo larvario. Revista Salud Animal. 2013; 35(2):103108. http://scielo.sld.cu/pdf/rsa/v35n2/rsa05213.pdf

6. Urdaneta-Fernadez M, Udameta Í, Parra A, Chacín E, Ramírez-Barrios R, Angulo-Cubillán F. Prevalencia y grado de infección de helmintos gastrointestinales en rebaños doble propósito del municipio Miranda del estado Zulia Venezuela. Revista de la Universidad de Zulia. 2011; 2(2):184-193. http://bdigital.ula.ve/storage/pdf/revluz/ v2n2/art08.pdf 
7. Soca M., Simoón L, Roque E. Árboles y nemátodos gastrointestinales en bovinos jóvenes: Un nuevo enfoque de las investigaciones. Pastos y Forrajes. 2007; 30(5):21-33. http://scielo.sld.cu/scielo.php?script=sci arttext\&pid=S0864-03942007000500002

8. Sixtos M. Procedimientos y técnica para la relación de estudios coproparasitoscópios. Virbac al día. 2011; 24: 6-7.

9. Axóncomunicación. Diagnóstico parasitológico a partir de muestras fecales (I). Cría y Salud. 2009; 28: 52-54. https:// www.academia.edu/31736033/Virbaccoprosparasitologia

10. Mathews P. Design of Experiments with MINITAB. Milwaukee, Wisconsin: ASQ Quality Press. 2004. https://www. academia.edu/23892705/Design of Experiments with MINITAB?auto=download

11. OIE. Principios y métodos de validación de las pruebas de diagnóstico de las enfermedades infecciosas. Manual acuático de la OIE. 2012; 1-20. https://www.oie.int/esp/normes/fmanual/1.1.02 VALIDATION.pdf

12. Cañez M, García A. Validación de un método analítico para la determinación de fósforo por espectrofotometría ultravioleta-visible. Revista de Ciencias Biológicas y de la Salud. 2015; 17(1):32-39. https://biotecnia.unison.mx/ index.php/biotecnia/article/viewFile/15/14

13. Faría J, Allara M, Arenas de Moreno L, Márquez E. Extracción y cuantificación de penicilina G en leche cruda por cromatografía líquida de alta resolución. Revista Científica. 2000; 10(3):212-221. https://produccioncientificaluz. org/index.php/cientifica/article/view/14705/14682 